This is an Accepted Manuscript version of the following article, accepted for publication in Comprehensive Psychiatry. Jessica Dere, Qiuping Tang, Xiongzhao Zhu, Lin Cai, Shuqiao Yao \& Andrew G. Ryder (2013): The cultural shaping of alexithymia: Values and externally oriented thinking in a Chinese clinical sample, Comprehensive Psychiatry, https://doi.org/10.1016/j.comppsych.2012.10.013. It is deposited under the terms of the Creative Commons Attribution-NonCommercial-License (http://creativecommons.org/licenses/by-nc/4.0/), which permits non-commercial re-use, distribution, and reproduction in any medium, provided the original work is properly cited

(c) (1) (3)

\title{
The cultural shaping of alexithymia: Values and externally oriented thinking in a Chinese clinical sample
}

\author{
Jessica Dere ${ }^{a, 1}$, Qiuping Tang ${ }^{b}$, Xiongzhao Zhu ${ }^{c}$, Lin Cai ${ }^{c}$, Shuqiao Yao ${ }^{c}$, Andrew G. Ryder ${ }^{a, d, *, \text { and } 1}$ \\ ${ }^{a}$ Concordia University \\ ${ }^{b}$ Third Xiangya Hospital \\ Central South University \\ ${ }^{c}$ Second Xiangya Hospital \\ Central South University \\ ${ }^{d}$ Lady Davis Institute and SMBD-Jewish General Hospital
}

\begin{abstract}
Objective: Alexithymia is a multi-faceted personality construct characterized by difficulties in identifying and describing emotional states. Originally based on observations of American psychosomatic patients, the construct is now studied in a variety of cultural contexts. However, few studies have critically examined alexithymia from a cultural perspective. Dere et al. [1] recently found support for the hypothesis that one alexithymia component - externally oriented thinking (EOT) - is linked to cultural values, among Euro-Canadian and Chinese-Canadian students. The current study examines this association in a Chinese clinical sample.

Methods: Outpatients presenting at three hospital-based psychology clinics in Hunan province, China $(\mathrm{N}=268)$ completed a structured clinical interview and self-report measures of alexithymia and cultural values. All participants endorsed clinically significant levels of depressed mood, anhedonia, and/or fatigue.

Results: As expected, EOT was negatively predicted by Modernization and Euro-American values. Two other alexithymia components, difficulty identifying feelings and difficulty describing feelings, were unrelated to cultural values.

Conclusion: These findings suggest that cultural variations in the importance placed on emotional experience must be taken into account in cross-cultural alexithymia research. Such studies should also consider separately the specific components of alexithymia; failure to do so can lead to overestimation of alexithymia in groups where scores are driven by culturally-promoted EOT. (C)2013 Elsevier Inc. All rights reserved.
\end{abstract}

\section{Introduction}

The construct of alexithymia was formally introduced in the early 1970s, in an attempt to operationalize a set of clinical characteristics observed among North American psychosomatic patients. Interest in alexithymia has since expanded considerably, with the literature now encompassing a broad

*Corresponding author. Department of Psychology (PY-148), Concordia University, 7141 rue Sherbrooke O., Montréal (Québec) H4B 1R6, Canada. Tel.: +1 514848 2424x5285; fax: +1 514848 4523.

E-mail addresses: jessicadere@gmail.com (J. Dere), andrew.ryder@concordia.ca (A.G. Ryder).

1 Correspondence and reprint requests may be addressed to Jessica Dere or Andrew G. Ryder. Jessica Dere is now a post-doctoral fellow in the Social Aetiology of Mental Illness (SAMI) CIHR Training Program, Centre for Addiction and Mental Health/University of Toronto, Toronto, Ontario, Canada. set of research questions and populations. The consequence is that the alexithymia construct is now applied to cultural contexts that diverge considerably from the one in which it was originally developed, thereby increasing the possibility of misinterpretation. The current study provides a cultural examination of alexithymia among Chinese depressed outpatients that raises a more significant concern, beyond simple misinterpretation. We propose that one alexithymia component- externally oriented thinking - can be culturally promoted, and therefore its inclusion in the overarching alexithymia construct may lead to the pathologization of cultural differences in emotion norms.

Coined by Sifneos and his colleagues [2,3], the literal meaning of alexithymia is 'a lack of words for emotion'. The concept originated in Sifneos' observations of psychosomatic patients in Boston, who presented particular challenges in the context of psychoanalytic therapy, the predominant therapeutic approach of the day [4]. Four main features define this construct: (1) difficulty identifying feelings, 
and distinguishing them from physical sensations of emotional arousal; (2) difficulty describing emotions to others; (3) reduced imaginal capacities; and (4) an externally oriented thinking style, with an emphasis on external, concrete stimuli rather than inner emotions [5].

The introduction of valid and reliable measures has been critical to the advancement of the alexithymia literature in recent decades. The most frequently used and wellvalidated measure is the Twenty-item Toronto Alexithymia Scale (TAS-20) [6-9]. The TAS-20 contains three subscales: Difficulty Identifying Feelings (DIF), Difficulty Describing Feelings (DDF), and Externally Oriented Thinking (EOT). The measure demonstrates good psychometric properties across a range of samples (e.g., [7,8]). The measure has also been translated into at least 18 different languages, including Chinese, with general cross-cultural support for the three-factor structure and for the reliability of the total score and the DIF and DDF subscales $[9,10]$. However, the EOT subscale frequently shows poor internal reliability, particularly in nonEnglish speaking samples [e.g., 9-12].

Whereas a number of studies using the TAS-20 have focused on cross-cultural measurement issues (e.g., [10]), few studies have addressed conceptual questions from a cultural perspective. Alexithymia has, however, been the target of several culturally-based theoretical critiques, with authors highlighting the construct's historical and cultural roots [4,13]. In particular, alexithymia has been linked to 'Western' norms that emphasize individual emotional experience and the verbal expression of emotion, with the suggestion that this construct would not carry the same meaning or relevance in contexts with different emotion norms [13]. We propose that a cultural perspective is particularly pertinent to EOT, since this component concerns the importance placed on emotional experiences. The importance of emotions is strongly related to cultural values and varies substantially across cultural contexts (e.g., [14,15]).

Our focus on EOT is based on several converging theoretical observations and empirical findings. First, there is the consistently poor reliability of the EOT subscale in nonEnglish speaking samples. Although this is a psychometric problem, it also suggests potential conceptual problems cross-culturally. Second, EOT stands apart from DIF and DDF in that it is not defined as an emotional deficit but rather a thinking style that de-emphasizes emotion. Finally, Ryder et al. [16] examined alexithymia among Euro-Canadian and Chinese depressed outpatients, and found that higher alexithymia levels among the Chinese were explained by higher levels of EOT; neither DIF nor DDF showed a significant group difference. These authors proposed that EOT may be promoted in a Chinese context due to greater cultural emphasis on social harmony, relationships, and contextual factors, with relatively less emphasis on individual emotional experience [17]. This proposal fits with a substantial literature on cultural differences in emotional processes among those of Chinese versus Western European heritage (e.g., [18]), but requires empirical examination.

Building on this work, Dere et al. [1] sought to test the general hypothesis that EOT would be particularly associated with cultural values, in contrast to DIF and DDF, among Euro-Canadian and Chinese-Canadian undergraduate students. Three sets of cultural values were examined - modernization, Euro-American values, and Asian values. Replicating Ryder et al. [16], higher total TAS-20 scores among the Chinese-Canadians were explained by a significant group difference on EOT. As hypothesized, Dere et al. found that modernization and Euro-American values negatively predicted EOT in both groups, while values were unrelated to DIF or DDF. Furthermore, cultural values mediated the effect of group membership on levels of EOT, helping to 'unpack' the observed cultural difference.

Together, the results of Ryder et al.'s [16] and Dere et al.'s [1] studies suggest the need to further examine the association between alexithymia and values in a Chinese context. The current study investigates the cultural promotion of EOT in a sample of depressed Chinese outpatients. As alexithymia is primarily a psychiatric construct, it is instructive to examine our cultural predictions in a clinical sample. Furthermore, the current participants were recruited in the same region as the Chinese sample in Ryder et al. [16], but cover a wider range of sociodemographics, providing a valuable sample in which to continue this line of research. Based on our theoretical expectations and previous findings, we examined the following hypotheses: (1) of the three TAS-20 subscales, only EOT would be predicted by cultural values; and (2) EOT would be negatively predicted by modernization and by Euro-American values. Although we would expect EOT to be positively predicted by Asian values on a theoretical basis, Dere et al. [1] found that Asian values did not predict EOT; therefore, we did not formulate a specific hypothesis regarding Asian values.

\section{Methods}

\subsection{Sites}

Data were collected at three sites in Hunan province, in south-central China, as part of a larger project examining cultural variations in depressive symptom presentation. The use of multiple sites allowed for the recruitment of both urban and rural Chinese outpatients, in contrast to the predominance of urban samples in previous studies. The first two sites were the Psychology Clinics at the Second $\mathrm{Xi}$ angya Hospital and the Third Xiangya Hospital, located in Changsha, the capital of Hunan; both are affiliated with Central South University, and serve the urban catchment area of Changsha along with its immediate suburbs. The third site 
was the Psychology Clinic at the Fourth Hospital of Huaihua; this hospital serves a rural catchment area approximately 400 $\mathrm{km}$ from Changsha. At all sites, clinical outpatients presenting for mental health services were eligible to participate in the research project. Potential participants were immediately excluded if they had a history of psychosis, mania, or neurocognitive deficits. Eligible patients were referred by their clinicians to trained research assistants; such patients then received information about the project and were informed that nonparticipation would not affect their care. All participants provided written informed consent. The study received ethical approval at all involved institutions.

\subsection{Participants}

A total of 308 outpatients participated in the project, which involved the completion of a structured clinical interview and a battery of self-report questionnaires. The interview was based on the mood disorder module of the Structured Clinical Interview for DSM-IV, Patient Version [19], with modifications for cross-cultural use similar to those described by Ryder et al. [16]. Most notably, questions were added based on the most recent version of the Chinese Classification of Mental Disorders (CCMD-3) [20]. Depression criteria unique to CCMD-3 were incorporated into the interview, and an additional section was developed based on the diagnostic criteria for neurasthenia - a disorder often seen as Chinese-specific, which overlaps with depression and is characterized by persistent mental and physical fatigue. Patients were included in the study if they endorsed at least one of the core symptoms of depression or neurasthenia as defined across the Diagnostic and Statistical Manual of Mental Disorders (4th ed.; DSM-IV) [21] and the CCMD-3 systems; these symptoms consist of clinically significant depressed mood, anhedonia, and fatigue. This approach helped to address the problems associated with using strict diagnostic categories as inclusion criteria, as such diagnoses are culturally defined and can mask cultural variation [16]. For the current study, participants were eliminated if: (a) they were younger than 18 or older than 65 ; (b) they were missing data on the self-report measures of interest; or (c) they did not endorse at least one of the core symptoms of depression or neurasthenia on the structured interview. These post hoc exclusion criteria resulted in the elimination of 2 participants due to age, 4 participants due to missing questionnaire data, and 34 participants based on the clinical symptoms criteria. Sample demographics for the final sample $(\mathrm{N}=268)$ can be found in Table 1.

\subsection{Measures}

Participants completed a series of self-report questionnaires assessing psychological distress, emotion regulation, and cultural variables. All measures were presented using the simplified Chinese character set. Only the four measures relevant to the current study will be discussed here. Alexithymia was assessed using the TAS-20. Following Dere et al. [1], cultural values were assessed in two ways: (1) a bipolar contrast of modernization versus traditionalism using the Modernization scale (MOD) of the Chinese Personality Assessment Inventory (CPAI) [22]; and (2) a bidimensional approach to cultural values measurement using the European American Values Scale for Asian AmericansRevised (EAVS-AA-R) [23] and the Asian Values ScaleRevised (AVS-R) [24] to measure EuroAmerican and Asian values, respectively.

\subsubsection{TAS-20}

The TAS-20 [6] is a 20-item measure of alexithymia with three subscales, as discussed earlier. A total score is computed across all items, after recoding reversed items. Subscale scores are calculated by summing the corresponding item scores for each subscale: DIF ( 7 items), DDF (5 items), and EOT (8 items). A previously validated Chinese version of the TAS-20 was used [10].

\subsection{2. $M O D$}

The MOD scale of the CPAI [22] is a 15-item measure of modern as opposed to traditional values and beliefs; it was constructed as part of the larger CPAI questionnaire, an indigenous Chinese measure of personality. A single mean score is computed for the MOD scale, after recoding reversed items; higher scores reflect endorsement of liberal values and a rejection of traditional beliefs and practices, while lower scores reflect greater endorsement of traditional beliefs and practices [25]. The original Chinese version of the scale was used.

\subsection{3. $E A V S-A A-R$ and $A V S-R$}

Both the EAVS-AA-R [23] and the AVS-R [24] are 25item measures of adherence to particular sets of cultural values. The EAVS-AA-R assesses values commonly adhered to in European American or 'Western' cultural contexts, such as individual achievement and personal autonomy. The AVS-R assesses values common in East and Southeast Asian cultural contexts, such as filial piety and collectivism. It is worth noting that the items on both measures are phrased as general values statements, and do not include specific cultural references. For both measures, a single mean score is computed after recoding reversed items. The Chinese versions of these measures were based on translations obtained from one of the measures' authors, B. S. K. Kim (personal communication, August 30, 2007), with minor modifications made for the local Chinese context. 
Table 1

Sample demographics $(N=268)$.

\begin{tabular}{lcc}
\hline & Percentage & Mean (SD) \\
\hline Sex (\% female) & $59.0 \%$ & $34.3(11.9)$ \\
Age (years) & & \\
Site & $37.3 \%$ & \\
Second Xiangya Hospital, Changsha & $19.4 \%$ & \\
Third Xiangya Hospital, Changsha & $43.3 \%$ \\
Fourth Hospital, Huaihua & $32.8 \%$ \\
Grew up in an urban setting ${ }^{\text {a }}$ & $67.5 \%$ \\
Currently living in an urban setting & \\
Education & & \\
Did not complete elementary school & $5.2 \%$ \\
Completed elementary school & $6.3 \%$ \\
Completed secondary school & $34.7 \%$ \\
Vocational training & $23.1 \%$ \\
Undergraduate degree & $27.2 \%$ \\
Doctorate or professional degree & $3.4 \%$ \\
\hline
\end{tabular}

${ }^{\text {a }}$ Urban setting was defined as a large or small city, or a suburb of a large city.

\section{Results}

\subsection{Data cleaning}

For each measure, univariate outliers were identified and brought in to within \pm 3 standard deviations from the mean, based on the method proposed by Tabachnick and Fidell [26].

\subsection{Scale reliability}

Table 2 displays Cronbach's alpha coefficients for all measures, along with descriptive statistics. The total TAS-20 and the DIF and DDF subscales all showed good internal consistency. As in previous studies, the EOT subscale showed the lowest reliability of the TAS-20 subscales. However, the reliability was particularly poor in this sample. Examination of the item-total correlations for this subscale revealed that there were no items whose removal would increase the sub- scale's internal consistency. The AVS-R and MOD showed good reliability, while the EAVS-AA-R showed low reliability. A reduced version of the EAVS-AAR was computed, after dropping five items that displayed negative item-total correlations in the reliability analysis; this reduced measure showed improved reliability, with a Cronbach's alpha of 0.57. All subsequent analyses were conducted with both the original and reduced versions of the EAVS-AA-R, with a nearly identical pattern of results; results using the full original measure are reported here.

\subsection{Regression analysis}

To examine our hypotheses regarding the prediction of EOT, DIF, and DDF by cultural values, multiple linear regression analyses were conducted. Following Dere et al. [1], one set of regression analyses examined MOD while a sec- 
Table 2

Scale reliability and descriptive statistics.

\begin{tabular}{lcccr}
\hline & $\alpha$ & Inter-item $\mathrm{r}$ & $\mathrm{M}$ & $\mathrm{SD}$ \\
\hline TAS-20 & 0.80 & 0.15 & 54.97 & 11.01 \\
DIF & 0.84 & 0.42 & 19.07 & 6.25 \\
DDF & 0.70 & 0.32 & 14.08 & 4.29 \\
EOT & 0.27 & 0.05 & 21.81 & 3.56 \\
MOD & 0.78 & 0.19 & 9.40 & 3.39 \\
EAVS-AA-R & 0.41 & 0.03 & 1.58 & 0.19 \\
AVS-R & 0.73 & 0.10 & 1.77 & 0.27 \\
\hline
\end{tabular}

TAS $-20=$ Twenty-item Toronto Alexithymia Scale. DIF $=$ Difficulty Identifying Feelings. DDF $=$ Difficulty Describing Feelings. EOT $=$ Externally Oriented Thinking. $\mathrm{MOD}=$ Modernization scale. EAVS-AA-R $=$ European American Values Scale for Asian Americans-Revised. AVS-R = Asian Values Scale-Revised.

ond set examined the EAVS-AA-R and AVS-R together. This approach allowed for internal replication of our results regarding the association between values and alexithymia. For each regression analysis, one TAS-20 subscale was entered as the dependent variable, age, sex, and current urban versus rural status were entered as predictors in the first step, the other two TAS-20 subscales were entered in the second step, and the relevant cultural values score(s) was(were) entered in the third step.

As hypothesized, EOT was predicted by cultural values. MOD significantly negatively predicted EOT, $\beta=-0.23$, $p<.01$; the addition of MOD to the model resulted in a significant $R^{2}$ ch of $0.04, F(1,261)=11.12, p<.01$. In the second regression model, the EAVS-AA-R also significantly negatively predicted EOT, $\beta=-0.26, p<.001$, though the AVS-R did not account for unique variance in EOT, $\beta=0.03$, $n s$. The addition of the EAVS-AA-R and AVS-R to the model resulted in a significant $R^{2}$ ch of $0.06, F(2,260)=9.02, p<.001$. These findings support our hypotheses.

Also in line with our first hypothesis, neither DIF nor DDF was significantly predicted by cultural values. MOD did not significantly predict DIF or DDF, $\beta \mathrm{s}=-0.02$ and $0.07, n s$, and neither did the EAVS-AA-R, $\beta=-0.02$ and -0.03 , ns, or AVS-R, $\beta \mathrm{S}=0.02$ and $-0.02, n s$. The addition of cultural values did not result in a significant $R^{2}$ ch in any of the models predicting DIF or DDF.

To help address the statistical concerns associated with examining null hypotheses, power calculations for multiple regression were conducted. When examining the prediction of DIF or DDF by cultural values, the current study had sufficient power $(>.80)$ to detect a minimum $R^{2}$ ch of 0.013 in the MOD models and 0.016 in the EAVS-AA-R and AVS$\mathrm{R}$ models. These calculations bolster our interpretation of the DIF and DDF results, though the possibility remains that there are real, albeit small, effects for these variables.

\subsection{Structural equation modeling}

In light of the very low reliability of the EOT subscale in the current sample, the planned multiple regression analyses were followed up with structural equation modeling (SEM). This technique provides the advantage of explicitly taking measurement error into account [27]. We constructed two models, one to test the relation between MOD and EOT (Fig. 1), and the other to test the relation between the EAVSAA-R and AVS-R and EOT (Fig. 2). The technique of parceling was used to create measured variables for the four constructs of interest - EOT, MOD, EAVS-AA-R, and AVS-R - allowing EOT and the cultural values to be specified as latent variables. As suggested in the literature [28], three parcels were constructed for each latent variable; in each case, the items from the relevant measure were randomly selected without replacement to construct the parcels. SEM analyses were conducted using Mplus, version 5 [29], and maximum likelihood estimation. Five fit indices were employed to assess 'goodness-of-fit': model chi square $\left(\chi^{2}\right)$, chi square/degrees of freedom ratio $\left(\chi^{2} / \mathrm{df}\right)$, Comparative Fit Index (CFI), Root 


\section{Figure 1}

Structural equation model of Modernization predicting Externally Oriented Thinking. Values indicate standardized regression weights. All paths are significant at $<.01$. MOD=Modernization scale. EOT=Externally Oriented Thinking.

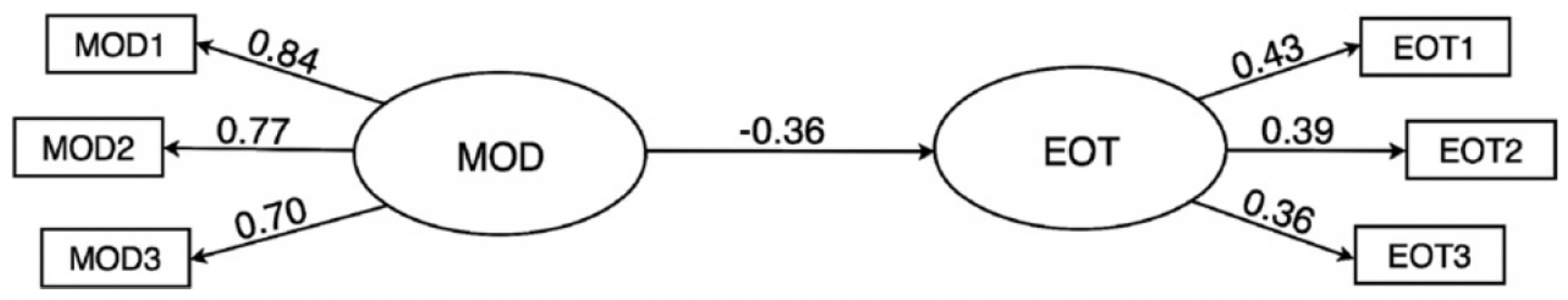

Mean Square Error of Approximation (RMSEA), and Standardized Root Mean Square Residual (SRMR). Overall fit for these indices was evaluated using the following criteria [30-32]: non-significant $\chi^{2}$; ratio of $\chi^{2} / \mathrm{df}$ adequate if $<5.0$ and good if $<2.0$; CFI adequate if $>0.90$ and good if $>0.94$; RMSEA adequate if $<0.10$ and good if $<0.05$; and SRMR adequate if $<0.08$ and good if $<0.05$.

The model testing the direct effect of Modernization on EOT showed good fit $\left(\chi^{2}=9.40, \mathrm{df}=8, p=0.31\right.$; $\chi^{2} / \mathrm{df}=1.18 ; \mathrm{CFI}=1.0 ; \mathrm{RMSEA}=0.03,90 \% \mathrm{CI}$ from 0 to 0.08 ; SRMR $=0.03$ ). All proposed pathways were significant, as shown in Fig. 1.

The model testing the direct effect of Euro-American and Asian values on EOT showed acceptable fit $\left(\chi^{2}=50.32\right.$, $\mathrm{df}=24, p=0.001 ; \chi^{2} / \mathrm{df}=2.10 ; \quad \mathrm{CFI}=0.92 ; \mathrm{RMSEA}=0.06$, $90 \%$ CI from 0.04 to 0.09; SRMR=0.05). Similar to the regression results, Euro-American values negatively predicted EOT but the proposed pathway from Asian values to EOT was not significant $(\beta=-0.14, n s)$. All remaining pathways were significant (see Fig. 2). The results of both SEM models replicate those from the multiple regression analyses predicting EOT.

\section{Discussion}

The current results provide strong support for our hypotheses. As expected, EOT was negatively predicted by both Modernization and Euro-American values. Furthermore, neither DIF nor DDF was significantly predicted by values, in line with our proposal that EOT is particularly shaped by cultural context. While the expected nonsignificant association between cultural values and DIF and DDF represents an attempt to evaluate null hypotheses, the pattern of results fits well with our theoretical model. Our results also replicate those of Dere et al. [1].

A particular strength of this study is the sample. Depressed outpatients are a pertinent group for this research, in light of the frequently examined link between alexithymia and depression (e.g., [33]). It is also noteworthy that our sample includes both urban and rural outpatients, likely capturing a wider range of cultural values, educational experiences, and socioeconomic status than the majority of previously studied Chinese clinical samples. This range of sociodemographics is particularly valuable in cultural research, enhancing the generalizability of our findings.

Our results hold important implications both for alexithymia research and for the cross-cultural study of emotion and emotional distress. In line with other recent work, our findings highlight the need to examine the components of alexithymia separately. Several authors have called for such an approach (e.g., [34]) in light of differential effects of the TAS-20 subscales on variables including autonomic reactivity [34], emotion word recall [35], and depressed mood [33]. Unfortunately, many studies do not examine the individual components. Based on the current findings, failure to do so may well lead to the overestimation of alexithymia in groups where TAS-20 scores are driven by culturallypromoted EOT. As high alexithymia levels are generally understood to reflect emotional deficits, such overestimation may result in the pathologization of groups whose emotionrelated norms and values diverge from those of the 'Western' groups most commonly used in alexithymia research.

The current findings, along with those of Dere et al. [1], point towards two possibilities regarding the place of EOT within the alexithymia construct. One possibility is that EOT is difficult to translate cross-culturally, both linguistically and conceptually. This would suggest that wording and content changes to current measures could improve the assessment and interpretation of EOT across cultural contexts. A second, more radical possibility is that EOT may be driven by different sets of factors in different cultural contexts. High levels of EOT are likely to be associated with high levels of DIF and DDF across a wide range of cultural contexts, since people who struggle to identify and describe their emotions are likely to focus outwards rather than inwards. In Chinese and other cultural contexts that foster an emphasis on external rather than internal experiences, however, adherence to 
Figure 2

Structural equation model of Euro-American and Asian values predicting Externally Oriented Thinking. Values indicate standardized regression weights. All reported paths are significant at $<.01$. EAVS-AA-R=European American Values Scale for Asian Americans-Revised. AVS-R=Asian Values Scale-Revised. EOT=Externally Oriented Thinking.

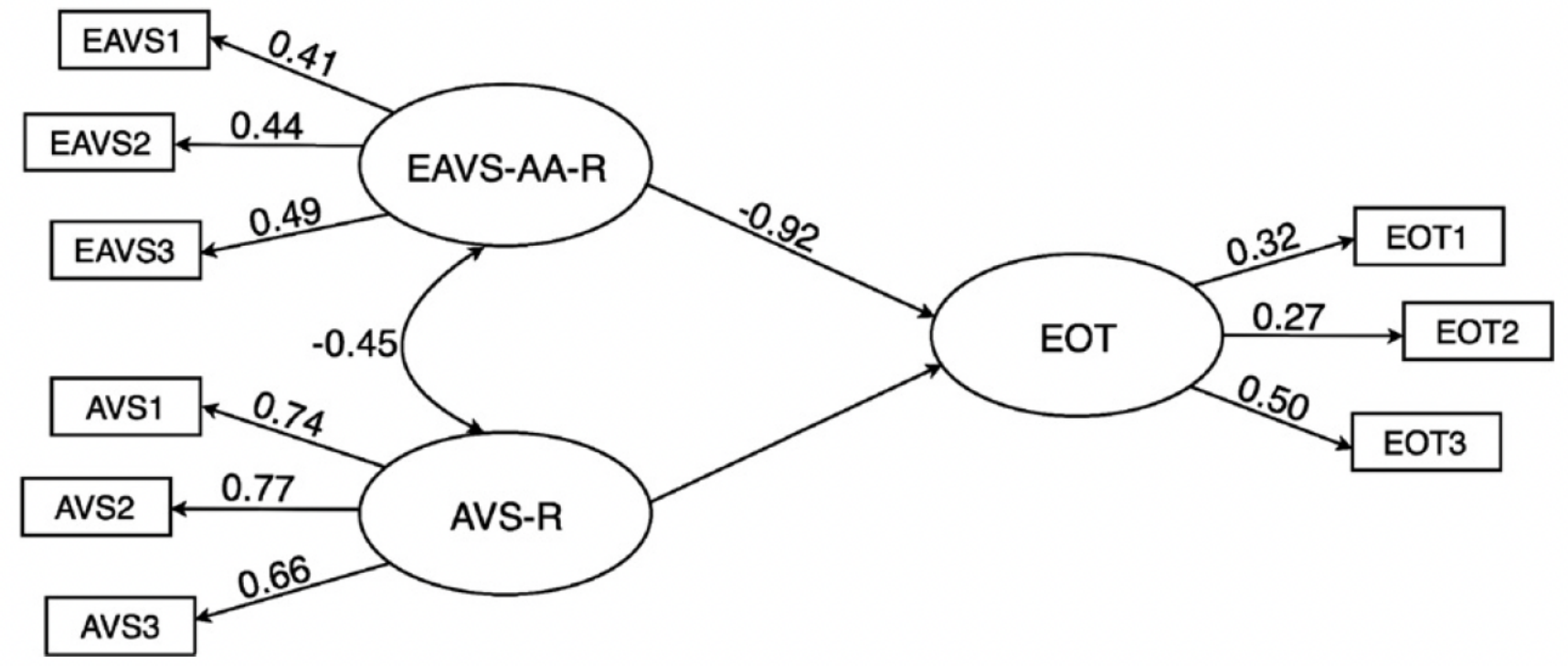

local emotional norms provides a second possible reason for higher levels of EOT. North American and other cultural contexts that promote attention to internal emotional experience and presentation of that experience to others do not provide this second pathway to EOT; indeed, diverging from local emotional norms by being externally focused may indicate psychopathology.

Although this proposal is currently a conceptual one, which requires direct empirical examination, Coffey et al. [36] provide findings that may support it. These authors conducted a factor analysis with the subscales of the TAS-20 and measures of emotional intelligence and mood awareness. They found two overarching factors, which they termed 'attention to emotions' and 'clarity of emotions'. EOT loaded more strongly on the attention factor, which the authors framed as a personality dimension, whereas DIF and DDF loaded on the clarity factor, which was framed as an aptitude. Drawing on our conceptual model, we would expect to find cultural differences in the emphasis placed on emotions (attention), but not in emotion-related deficits (clarity).

The finding that EOT is related to cultural values also has potential implications for the study of cultural variations in the presentation of emotional distress, particularly among Chinese and 'Western' samples. Ryder et al. [16] found that EOT partially mediated the relation between group membership and somatic symptom reporting among Chinese and Euro-Canadian outpatients, helping to partly explain the higher levels of somatic symptoms in the Chinese group. There are reasons to expect that cultural differences in the importance placed on emotional experience might be associated with differences in the experience and reporting of psychiatric symptoms [37,38]. Future research should seek to further examine the links between cultural values, EOT, and symptom presentation. In light of the important role that stigma can play in the presentation of mental illness (e.g., $[39,40])$, future work should also examine the associations between stigma and this set of variables.

Our study has several important limitations. Most critical is the low reliability of the EOT subscale, which simultaneously suggests the need for closer examination of this construct while making it difficult to test conceptual research questions. The use of parceling and SEM analyses helped to address this problem to a certain extent by directly accounting for measurement error. However, parceling remains controversial within the SEM literature [28]. Future studies should include multiple measures related to EOT and additional cultural variables, both to extend the current research and to construct an SEM model that does not require parceling. In addition, the values measures used in the current study each capture a broad set of cultural values. It would be desirable for future studies to incorporate measures that assess more narrow sets of values, including more contextspecific values as well as values about emotion (e.g., [41]). It is also worth noting that the current participants were seek- 
ing psychiatric services, which may differentiate them in important ways from other distressed Chinese groups, such as those who primarily use traditional medicine. Finally, the fact that all of the measures used in the current study were self-report represents an important limitation, though one shared by much alexithymia research. The Toronto Structured Interview for Alexithymia (TSIA; [42]) was recently developed by the authors of the TAS-20, and has been validated in at least two languages other than English [43,44]. Replication of the current findings using multiple assessment methods, including the TSIA, is an important future research direction.

The current research demonstrates the value of examining clinical constructs from a cultural perspective. Given the particular socio-cultural roots of alexithymia, it is perhaps especially important to critically examine this construct in a range of cultural settings, to help prevent the confounding of cultural variation in emotion-related values with emotional deficits. While our hypotheses and interpretations are framed within cultural theory and research, it is hoped that this work can inform a more nuanced understanding of alexithymia more generally.

\section{Acknowledgment}

We acknowledge the work of the translators and interviewers who contributed to the present study - Luo Dan, Zhong Mingjie, Liao Sha, and Jenny Teng. The current study was facilitated by a doctoral fellowship from the Fonds de la recherche en santé du Québec to Jessica Dere, and a Canadian Institutes of Health Research standard operating grant to Andrew G. Ryder. This research was conducted as part of Jessica Dere's doctoral dissertation at Concordia University, supervised by Andrew G. Ryder.

\section{References}

[1] Dere J, Falk CF, Ryder AG. Unpacking cultural differences in alexithymia: the role of cultural values among Euro-Canadian and Chinese-Canadian students. J Cross Cult Psychol 2012;43:1297-312.

[2] Sifneos PE. Prevalence of alexithymic characteristics in psychosomatic patients. Psychother Psychosom 1973;22:255-62.

[3] Nemiah JC, Freyberger H, Sifneos PE. Alexithymia: a view of the psychosomatic process. In: Hill OW, editor. Modern trends in psychosomatic medicine. London: Butterworths; 1976. p. 430-9.

[4] Prince R. Alexithymia and verbal psychotherapies in cultural context. Transcult Psychiat 1987;24:107-18.

[5] Taylor GJ. Recent developments in alexithymia theory and research. Can J Psychiat 2000;45:134-42.
[6] Bagby RM, Parker JDA, Taylor GJ. The twentyitem Toronto Alexithymia Scale I: item selection and cross-validation of the factor structure. J Psychosom Res 1994;38:23-32.

[7] Bagby RM, Taylor GJ, Parker JD. The twenty-item Toronto Alexithymia Scale II: convergent, discriminant, and concurrent validity. J Psychosom Res 1994;38:33-40.

[8] Parker JDA, Taylor GJ, Bagby RM. The 20-item Toronto Alexithymia Scale III. Reliability and factorial validity in a community population. J Psychosom Res 2003;55:269-75.

[9] Taylor GJ, Bagby RM, Parker JDA. The 20-item Toronto Alexithymia Scale IV. Reliability and factorial validity in different languages and cultures. J Psychosom Res 2003;55:277-83.

[10] Zhu X, Yi J, Yao S, Ryder AG, Taylor GJ, Bagby RM. Cross-cultural validation of a Chinese translation of the 20-item Toronto Alexithymia Scale. Compr Psychiatry 2007;48:489-96.

[11] Bressi C, Taylor G, Parker J, Bressi S, Brambilla V, Aguglia E, et al. Cross validation of the factor structure of the 20-item Toronto Alexithymia Scale: an Italian multicenter study. J Psychosom Res 1996;41:551-9.

[12] Moriguchi Y, Maeda M, Igarashi T, Ishikawa T, Shoji $\mathrm{M}$, Kubo C, et al. Age and gender effect on alexithymia in large, Japanese community and clinical samples: a crossvalidation study of the Toronto Alexithymia Scale (TAS-20). Biopsychosoc Med 2007;1:7.

[13] Kirmayer LJ. Languages of suffering and healing: alexithymia as a social and cultural process. Transcul Psychiat Res Rev 1987; 24:119-36.

[14] Markus HR, Kitayama S. Culture and the self: implications for cognition, emotion, and motivation. Psychol Rev 1991;98:224-53.

[15] Eid M, Diener E. Norms for experiencing emotions in different cultures: inter- and intranational differences. $\mathbf{J}$ Pers Soc Psychol 2001;81:869-85.

[16] Ryder AG, Yang J, Zhu X, Yao S, Yi J, Heine SJ, et al. The cultural shaping of depression: somatic symptoms in China, psychological symptoms in North America? J Abnorm Psychol 2008;117:300-13.

[17] Heine SJ. Self as cultural product: an examination of East Asian and North American selves. J Pers 2001;69:881906.

[18] Mesquita B, Leu J. The cultural psychology of emotion. In: Kitayama S, \& Cohen D, editors. Handbook of cultural psychology. New York: The Guilford Press; 2007. p. 734-59.

[19] First MB, Spitzer RL, Gibbon M, Williams JBW. Structured clinical interview for DSM-IV axis I disorders, research version, patient edition (SCID-I/P). New York: Biometrics Research, New York State Psychiatric Institute; 1997. 
[20] Chinese Psychiatric Society. The Chinese classification of mental disorders. 3rd ed. (CCMD-3) Shandong: Shandong Publishing House of Science and Technology; 2001.

[21] American Psychiatric Association. Diagnostic and statistical manual of mental disorders. 4th ed. Washington, DC: Author; 1994.

[22] Cheung FM, Leung K, Fan R, Song WZ, Zhang JX, Zhang JP. Development of the Chinese Personality Assessment Inventory (CPAI). J Cross Cult Psychol 1996;27:18199.

[23] Hong S, Kim BSK, Wolfe MM. A psychometric revision of the European American Values Scale for Asian Americans using the Rasch model. Meas Eval Couns Dev 2005;37:194-207.

[24] Kim BSK, Hong S. A psychometric revision of the Asian Values Scale using the Rasch model. Meas Eval Couns Dev 2004;37:15-27.

[25] Cheung FM, Kwong JYY, Zhang J. Clinical validation of the Chinese Personality Assessment Inventory. Psychol Assess 2003;15:89-100.

[26] Tabachnick BG, Fidell LS. Using multivariate statistics. 4th ed. Needham Heights, MA: Allyn \& Bacon; 2001.

[27] Kline RB. Principles and practice of structural equation modeling. 3rd ed. New York, NY: The Guilford Press; 2011.

[28] Little TD, Cunningham WA, Shahar G. To parcel or not to parcel: exploring the question, weighing the merits. Struct Equ Model 2002;9:151-73.

[29] Muthén LK, Muthén BO. Mplus user's guide. 5th ed. Los Angeles, CA: Muthén \& Muthén; 2007.

[30] Bryne BM. Structural equation modeling with EQS and EQS/ Windows: basic concepts, applications, and programming. Thousand Oaks, CA: Sage Publications; 1994.

[31] Ullman JB. Structural equation modeling. In: Tabachnick BG, \& Fidell LS, editors. Using multivariate statistics. New York: Harper Collins College Publishers; 1996. p. 708-819.

[32] Hu L, Bentler PM. Cutoff criteria for fit indexes in covariance structure analysis: conventional criteria versus new alternatives. Struct Equ Model 1999;6:1-55.

[33] Saarijärvi S, Salminen JK, Toikka TB. Alexithymia and depression: a 1-year follow-up study in outpatients with major depression. J Psychosom Res 2001;51:729-33.

[34] Pollatos O, Werner NS, Duschek S, Schandry R, Matthias E, TrautMattausch E, et al. Differential effects of alexithymia subscales on autonomic reactivity and anxiety during social stress. J Psychosom Res 2011;70:525-33.

[35] Luminet O, Vermeulen N, Demaret C, Taylor GJ, Bagby RM. Alexithymia and levels of processing: evidence for an overall deficit in remembering emotion words. J Res Pers 2006;40:713-33.

[36] Coffey E, Berenbaum H, Kerns JG. The dimensions of emotional intelligence, alexithymia, and mood awareness: associations with personality and performance on an emotional stroop task. Cognit Emotion 2003;17:671-9.

[37] Ryder AG, Ban LM, Chentsova-Dutton YE. Towards a cultural- clinical psychology. Soc Pers Psychol Compass 2011;5:960-75.

[38] Ryder AG, Chentsova-Dutton YE. Depression in cultural context: 'Chinese somatization', revisited. Psychiat Clin N Am 2012;35:15-36.

[39] Raguram R, Weiss MG, Channabasavanna SM, Devins GM. Stigma, depression and somatization in South India. Am J Psychiatry 1996;153:1043-9.

[40] Yang LH, Kleinman A, Link BG, Phelan JC, Lee S, Good B. Culture and stigma: adding moral experience to stigma theory. Soc Sci Med 2007;64:1524-35.

[41] Mauss IB, Butler EA, Roberts NA, Chu A. Emotion control values and responding to anger provocation in Asian-American and European-American individuals. Cognit Emotion 2010;24:1026-43.

[42] Bagby RM, Taylor GJ, Parker JD, Dickens SE. The development of the Toronto Structured Interview for Alexithymia: item selection, factor structure, reliability and concurrent validity. Psychother Psychosom 2006;75:25-39.

[43] Caretti V, Porcelli P, Solano L, Schimmenti A, Bagby RM, Taylor GJ. Reliability and validity of the Toronto Structured Interview for Alexithymia in a mixed clinical and nonclinical sample from Italy. Psychiat Res 2011;187:432-6.

[44] Grabe HJ, Löbel S, Dittrich D, Bagby RM, Taylor GJ, Quilty LC, et al. The German version of the Toronto Structured Interview for Alexithymia: factor structure, reliability, and concurrent validity in a psychiatric patient sample. Compr Psychiatry 2009;50:424-30. 